FORMATION Formation emploi

Revue française de sciences sociales

99 | juillet-septembre 2007

Les usages sociaux de la compétence

\title{
La gestion des émotions aux pompes funèbres, une compétence reconnue?
}

Coping with emotion at funeral undertakers' firms. Is this competence properly recognized?

Trauerbegleitung durch Bestattungsunternehmen. Eine anerkannte Kompetenz?

Julien Bernard

\section{(2) OpenEdition}

Journals

Édition électronique

URL : http://journals.openedition.org/formationemploi/1472

DOI : 10.4000/formationemploi.1472

ISSN : 2107-0946

Éditeur

La Documentation française

Édition imprimée

Date de publication : 1 juillet 2007

Pagination : 61-74

ISSN : 0759-6340

\section{Référence électronique}

Julien Bernard, «La gestion des émotions aux pompes funèbres, une compétence reconnue ? »,

Formation emploi [En ligne], 99 | juillet-septembre 2007, mis en ligne le 30 septembre 2009, consulté le 30 octobre 2020. URL : http://journals.openedition.org/formationemploi/1472 ; DOI : https://doi.org/ 10.4000/formationemploi.1472 


\section{DOSSIER}

\section{La gestion des émotions aux pompes funèbres, une compétence reconnue?}

Par Julien Bernard*

Les opérateurs des pompes funèbres travaillent dans un contexte sensible. Comment font-ils face aux émotions? Comment se protègent-ils tout en compatissant?

La gestion des émotions apparaît dès lors comme une compétence nécessaire.

Le travail des pompes funèbres connaît actuellement d'importantes transformations aux niveaux institutionnel et pratique. Deux lignes-forces - les conditions d'exercice de la profession et la définition du contenu des savoirs professionnels - orientent les discussions entre les acteurs du secteur et avec les autorités administratives. La qualité du service est au cœur des débats.

Un récent rapport d'information sur la législation funéraire au Sénat en témoigne (Sueur et Lecerf, 2006, pp. 45-50). Tout en encourageant les opérateurs funéraires à poursuivre les efforts développés ces dernières années, il considère que "les conditions d'habilitation ne garantissent pas la qualité des opérateurs funéraires », et recommande, sur ce point, de renforcer le contrôle de ces procédures et d'«améliorer la formation professionnelle des personnels». Le Rapport a en effet noté que la qualité des prestations était parfois fort insuffisante, " tant pour l'accueil des familles (absence de devis précis, mauvaise information des personnes endeuillées) que pour l'organisation concrète des obsèques (manque de décence du personnel, déroulement improvisé de la cérémonie) ». La profession funéraire doit être exercée, rappelle le Rapport, "avec toute la décence et les compétences que les circonstances exigent $»$.

\footnotetext{
* Julien Bernard est doctorant en sociologie (Équipe d'accueil identité et connaissance des territoires en mutation, Poitiers), altaché temporaire d'enseignement et de recherches à l'UFR (Unité de formation et de recherches) Sciences Humaines et Arts de l'université de Poitiers. Ses travaux se situent dans le domaine de la sociologie des émotions. Sa thèse, en cours, porte sur le travail du sentiment dans le travail des pompes funèbres. II est l'auteur notamment de "Les émotions dans la relation pompes funèbres-endeuillés. Une problématique de santé ", Face à Face Regards sur la santé, (2006), Revue électronique, $n^{\circ} 9$, Dossier «Émotions, corps et santé : gouverner par la parole? »
} 
Développer et garantir les compétences des opérateurs funéraires légitime l'étude du caractère très sensible de leurs situations de travail. Leurs clients, les familles endeuillées, sont plongées dans l'immédiat et la force de leurs émotions. Habitués, les opérateurs funéraires ont davantage de recul. Dans ce décalage, que le sociologue Hughes appelait le «drame social du travail» (Hughes, 1976), ils essaient d'adapter leur conduite et veillent à ne pas heurter la sensibilité de leurs clients, à faire preuve de moralité, à proposer un certain soutien.

La place des émotions dans cette relation asymétrique est primordiale. Les agents doivent se protéger tout en s'engageant dans la relation. Comme pour de nombreuses activités de service aux particuliers, la satisfaction des clients est considérée comme centrale. La plupart des entreprises proposent une prise en charge globale et surtout personnalisée des obsèques «au service des familles $»^{1}$. Prendre en compte l'état affectif des clients et offrir quelque chose d'eux-mêmes dans ces relations est, semble-til, souhaité par les autorités publiques, par les clients eux-mêmes, ainsi que par de nombreux professionnels soucieux de faire valoir leur compétence.

Partant du terrain, cet article s'inscrit dans le cadre d'une thèse de sociologie sur les émotions dans le travail des pompes funèbres. L'observation et la participation à ces activités, pendant dix mois en 2003-2004 et deux mois en 2006 dans une autre entreprise en tant qu' « agent d'exécution funéraire », conjuguée à de nombreuses discussions informelles avec des professionnels du funéraire ont permis d'une part, de découvrir la variété des pratiques et des savoirs professionnels déployés, d'autre part, de mettre à jour le poids des émotions, de soi ou d'autrui, sur les situations, mais aussi le travail accompli sur ces émotions. Parallèlement, des entretiens semi-directifs formels $(\mathrm{n}=8)$ et informels $(n=13)$ auprès de professionnels, sur les thèmes de l'entretien funéraire et de l'organisation des funérailles, ont confirmé l'importance de cet aspect : la gestion des émotions s'impose comme une nécessité.

\footnotetext{
1 Cf. article 1 du code de déontologie professionnelle de la Confédération des pompes funèbres et de la marbrerie, par exemple.
}

L'enjeu de la notion de compétence dans les pompes funèbres prend son sens au niveau de la professionnalisation du secteur. La lecture des revues du secteur, des documents professionnels ou de la législation, montre un groupe professionnel en structuration. Ainsi, la notion de compétence est différemment mobilisée et définie mais « la » compétence des opérateurs commence à être « objectivée ». La mise en place, en 2007, d'un nouveau CQP (Contrat de qualification professionnelle) pour les assistants funéraires, validable en VAE (Validation des acquis de l'expérience), donne lieu à un référentiel de compétences plus précis qu'auparavant. Néanmoins, une part du référentiel concerne directement des compétences relationnelles, de savoir-être, dont la définition est très floue, l'appréciation subjective et les conditions d'évaluation non précisées.

Comment les compétences des opérateurs funéraires peuvent-elles être définies, évaluées, jugées ? L'article invite à intégrer la question des émotions dans cette discussion. Il aborde, dans un premier temps, la manière dont les émotions au travail ont été étudiées par la sociologie, puis explore la place de celles-ci dans le travail concret des pompes funèbres.

Dans un second temps, la question de la compétence et de la formation professionnelle - posée par les acteurs du secteur comme par la Commission du Sénat - sera abordée à partir d'un rappel du contexte institutionnel des pompes funèbres, d'un état des lieux de la profession en matière de formation professionnelle, et par le discernement des usages différenciés de la notion de compétence selon les points de vue des acteurs du secteur.

Nous conclurons sur la nécessité d'objectiver les modes de jugement des compétences émotionnelles des assistants funéraires, dans le travail réel comme dans les évaluations qui en sont faîtes. Mais il conviendra d'inscrire cette notion en critique d'une réduction individualisante de l'intelligence émotionnelle et spécifier en quoi le travail émotionnel doit surtout aux configurations de l'interaction, à l'organisation collective du travail, à sa logistique et aux conditions sociales dans lesquelles il s'exerce. 
L'ART DE FAIRE AVEC LES ÉMOTIONS

\section{Travailler les émotions, une pénibilité nécessaire}

Le thème des émotions au travail a derrière lui une certaine tradition. Il connaît aujourd'hui un intérêt croissant, notamment en sciences de gestion et management. Le terrain du travail et de la santé ont été privilégiés. À partir des années 60-70, le terme de «sociologie des émotions» semble recouvrir un champ précis suite aux travaux de Goffman sur la «figuration» puis de Hochschild sur le «travail émotionnel » qui étudient les émotions dans une perspective interactionniste (dramaturgique et culturaliste). Leur point commun est de vouloir «sociologiser» les émotions elles-mêmes, en les considérant comme des produits des situations sociales. Les manières dont l'individu «négocie» avec ses propres émotions et celles dont il use pour identifier et juger celles des autres (Paperman et Ogien, 1995) ne sont pas négligées.

Selon Hochschild (1975), un travail est émotionnel s'il se déroule face à un public, si les membres de l'interaction expriment des émotions produisant un effet sur les autres participants, et si les employeurs peuvent exercer d'une manière ou d'une autre un « contrôle » des émotions des employés, au nom de la dimension émotionnelle de leur tâche. C'est un travail sur soi imposé conjointement par soi-même et par l'organisation pour (faire) exprimer les « règles du sentiment», les émotions appropriées, en situation de travail. Selon Shott, il agit comme un mécanisme de contrôle social qui focalise sur l'expérience déplaisante des sujets et remarque le décalage entre leur émotion réelle et l'émotion prescrite (Shott, 1979). Ce décalage produit du stress et accélère les stratégies de réponse comportementale ou cognitive qui, parfois, cependant, échouent et conduisent à l'étiquetage du sujet comme «émotionnellement déviant» (Thoits, in Kemper, 1990). Selon A. Soares, il peut être «intégrateur» (producteur d'amabilité), «dissimulateur » (neutralisation affective) ou «différenciateur 》 (distanciation) (Soares, 2002, p. 11)

D'autres auteurs ont évoqué une notion proche, celle de «travail du sentiment». A. Strauss emploie cette notion pour désigner «une des modalités de travail de soi et sur soi indissociable de l'articulation entre plusieurs lignes d'action dans un arrangement d'interaction » (Strauss, in Cefaï, 2003, p. 378, note 17). Il convient alors de distinguer émotions et sentiments. De l'avis de plusieurs, les émotions sont les manifestations des sentiments. Plus précisément, selon le philosophe P. Livet, elles sont « la résonance affective [...] d'un différentiel entre des traits perçus, imaginés ou pensés de la situation en cause et le prolongement de nos pensées [...] en cours [...] apprécié relativement à nos orientations affectives (désirs, préférences, sentiments, humeurs) » (Livet, 2002, p. 18). Les sentiments forment des dispositions activables, plus stables que le résultat de leur activation, les émotions. Le «différentiel » fonctionne alors comme un signal d'alarme.

Sur la santé des travailleurs, de nombreux auteurs ont souligné la difficulté, voire la souffrance, que le travail émotionnel pouvait causer (Dejours, 1993 ; Grosjean et Van de Weerdt, 2003). L'étude des relations professionnelles révèle la difficulté de trouver la «juste distance» d'avec l'interlocuteur (Loriol et Weller, 2002), surtout si le travail nécessite aussi de gérer les émotions des autres. Selon un document de la Fédération française de santé au travail, «l'assistant funéraire a une charge mentale importante, mais il doit toujours donner l'impression de disponibilité et de sérénité [...] le contact permanent avec des personnes endeuillées peut diminuer sa résistance psychologique ».

Le statut des émotions est encore mal circonscrit. Comme le remarque A. Jeantet (2002), on ne sait pas toujours si les émotions sont «objets» du travail (gestion des émotions des endeuillés, évitement du «piège de la compassion » (Goffman, 1968, pp. 380383)), « outils 》 du travail (aides à la prise de décision (Damasio, 1995)), « effets » du travail (compassion, répulsion, peur, etc. dont l'origine se situe dans la situation de travail) ou «acteurs » du travail (le travail des émotions versus le travail sur les émotions).

Dans le cas des pompes funèbres, d'après nos observations, elles sont les quatre à la fois. Le travail avec les émotions apparaît comme pénible et nécessaire. Il nécessite une «composition » de rôle adéquate que 
Trompette et Caroly appellent «compétence de gestion de l'interaction ». Garder le contrôle de la situation nécessite de trouver des techniques de gestion de soi. Cela est utile pour mieux pouvoir, ensuite, s'occuper des autres. Selon ces auteurs, l'intérêt d'en décrire la mise en œuvre en situation de travail réside dans la participation au débat social sur la définition des "modalités de construction des compétences en terme de gestion des savoirs professionnels. » (Trompette et Caroly, 2006). Pour nous rendre compte de ces dimensions, nous proposons d'examiner plus précisément le travail des pompes funèbres in situ.

\section{Composer un rôle dans des situations relationnellement complexes}

Cette gestion des savoirs professionnels s'exprime dans l'articulation entre les trois fonctions principales des opérateurs funéraires : conclure avec les familles l'organisation et les conditions de la prestation, coordonner le déroulement des diverses cérémonies de la mise en bière jusqu'à l'inhumation ou la crémation, et exécuter les prestations funéraires.

Coordination et personnalisation résonnent comme deux mots d'ordre. Ils se manifestent dans l'échange commercial, la préparation et l'accomplissement de la cérémonie. L'engagement subjectif dans la compréhension de chaque cas fait naître des émotions liées, d'une part au régime de la compassion et d'autre part à la tension de l'échange commercial. Leur maitrise suppose une articulation entre empathie et compétence d'orchestration.

Cette tension s'inscrit dans la relation de service proprement dite, qui débute lors de l'entretien des familles avec le conseiller. L'établissement de la confiance est difficile (Trompette et Boissin, 2004 a). Argent et sacré s'accordent mal et la première approche clientèle est primordiale. Le soupçon peut porter sur les «raisons» de la prise en charge des émotions de la clientèle, humaniste mais aussi voire (surtout?) économique. Si les opérateurs s'emploient, selon Trompette et Caroly, à « coconstruire " la demande avec le client, i.e. une «fourchette» de prix acceptable, le passage à la vente des produits funéraires demeure délicat. Selon un directeur d'agence interviewé, «il faut sentir si c'est une formalité pour eux d'enterrer leur grandpère ou leur grand-mère ou si c'est vraiment... avec beaucoup de peine et de là, il faut sentir quand le client est le plus disposé à acheter...» (directeur, 62 ans).

Par ailleurs, des changements sociologiques majeurs - urbanisation, éclatement des familles, rationalisation logistique de la prestation de service, individuation et déculturation du rapport à la mort etc. redéfinissent le rapport social à la mort et le rôle du professionnel du funéraire. Celui-ci est de plus en plus tenu de poursuivre deux objectifs différents : vendre et ritualiser. Or, malgré des évolutions récentes, les rituels funéraires demeurent très codifiés. Dès lors, les agents funéraires « orchestrent» aussi la ritualité des obsèques en observant un certain ritualisme, par une protocolarisation des démarches qui peut, parfois, entraîner une dépersonnalisation de la relation.

Entre personnalisation et conventions, meilleur sera l'entretien funéraire, meilleure sera la coordination ultérieure entre l'organisation des pompes funèbres et les volontés des familles. Cette entente est d'autant plus nécessaire que la coordination logistique des obsèques fait ensuite intervenir toute la gamme de professions du secteur, nécessaire à l'ensemble du service.

\section{Le contrôle des émotions : un savoir construit collectivement}

Les professionnels du funéraire s'emploient à calmer ou à rassurer les endeuillés. Mais selon leur poste dans l'entreprise, les situations auxquelles ils ont affaire et les spécificités de leur travail émotionnel varient.

L'entraide entre les différents postes est possible et les directeurs responsables essaient de protéger leurs employés des impacts émotionnels de manière appropriée. En effet, la secrétaire qui reçoit les quelques personnes proches du défunt pour l'entretien funéraire - dans un moment d'échange de paroles parfois intime - et l'équipe de porteurs qui exécute la prestation devant l'assistance des obsèques, ne travaillent pas dans les mêmes conditions ni ne sont exposées aux mêmes émotions. 


\section{Encadré 1}

\section{Description d'une procédure-type, de la prise de contact jusqu'à la fin du service}

- L'entretien funéraire. Le décès est constaté. L'assistant funéraire reçoit la famille. II doit obtenir les informations clés pour la suite de la procédure: Qui est mort? Où le corps repose-til ? Souhaite-ton une cérémonie religieuse? Choix d'une inhumation ou d'une crémation? II prend en charge diverses démarches et vend différents objets ou prestations.

- Le jour de la cérémonie, l'équipe des pompes funèbres se rend sur le lieu de repos du corps pour la "mise en bière ». Le corps du défunt est placé dans le cercueil et préparé par les agents d'exécution, parfois en présence de la famille. Le cercueil est ensuite fermé et transporté manuellement jusqu'au corbillard.

- La famille et les pompes funèbres forment cortège pour se rendre au lieu de culte, au cimetière ou au crématorium. La cérémonie peut être civile ou religieuse. Le cercueil est disposé sur des tréteaux entourés de fleurs et de plaques.

- L'inhumation ou la crémation. Lors d'un enterrement, le cercueil est descendu par un système de cordage. Lors d'une crémation, il est enfourné en back office par les agents du crématorium, et restitué à la famille plusieurs heures plus tard sous la forme d'une urne cinéraire.

$\mathrm{Au}$ cours de notre observation, nous nous sommes particulièrement intéressé à l'accomplissement des cérémonies, du fait de notre position de porteur. Deux types de moments-clés pour la gestion émotionnelle des pompes funèbres ont pu être repérés : ceux concernant la découverte de l'intensité de l'émotion des endeuillés et ceux relatifs à l'accomplissement des «passages » les plus délicats.

«Ils n'ont pas l'air trop peinés d'apparence» (porteur, 70 ans). Cette réflexion spontanée illustre la forme que peut prendre la première « évaluation » de l'état affectif des endeuillés. En effet, c'est en arrivant face au «public » que les agents vont mesurer la difficulté de leur travail à venir. Même si, selon certains d'entre eux, l'expérience a pu renforcer leur maîtrise de soi - «maintenant, ça ne me fait plus rien» (responsable d'agence, 53 ans) - ils font une différence importante entre les « gros » enterrements, où la charge émotionnelle est forte, et les autres. Les conseillers caractérisent leurs clients en utilisant les émotions comme indicateur afin d'adapter leur procédure, leur attitude ${ }^{2}$.

Les «transitions de phase» (Livet, 2002) (mise en bière, fermeture du cercueil, inhumation ou crémation) sont ensuite des instants très denses de ruptures symboliques entre le mort et le monde des vivants. Les endeuillés perçoivent dans leur interaction diffuse les signes de la réalisation du cours d'action et ses implications. Le mécanisme de l'émotion collective s'appuie sur cette réalisation de la réalité, ou, selon P. Dumouchel (1995), les «moments saillants de coordination collective ».

La régulation sociale de ces émotions résulte alors de l'adaptation conséquente des conduites. Le fait de travailler sous le regard du client accentue cette application maximale. Le "maître de cérémonie» est ici une personne ressource, un " acteur-expert», chargé de la mise en œuvre des compétences du personnel porteur de cercueil, à travers l'imposition de consignes précises dont le respect est censé garantir la qualité du service.

Ces deux exemples montrent le déploiement d'un savoir situé sur les émotions. L'effet de cette compétence possiblement «incorporée» (Leplat, 1997) s'observe dans les comportements des agents d'exécution. Ils mettent en place, par une concentration sur les tâches et une maîtrise des à-coups dans les mouvements - attention, gestes lents, pas anticipés la régulation de la charge émotionnelle. Autour du cercueil par exemple, ils réalisent un véritable ballet lors de la préparation du corps. Les compétences techniques, comme «savoir fermer le couvercle du cercueil» ou «le visser sans forcer», en sont les supports. De même, «savoir faire passer les cordes

2 Un peu à la façon des kinésithérapeutes, étudiés par P. Olry (2005), qui utilisent la douleur de leurs patients pour élaborer leurs «procédures » d'action et de soin. 


\section{Encadré 2 \\ Les fonctions des opérateurs}

\begin{tabular}{|c|c|}
\hline Niveau & Dénominations réglementaires et professionnelles \\
\hline 1 & $\begin{array}{l}\text { Les agents qui exécutent la prestation funéraire. } \\
\text { Porteurs et chauffeurs de véhicules funéraires; fossoyeurs; agents de crématorium; } \\
\text { agents de chambre funéraire. }\end{array}$ \\
\hline 2 et 3 & $\begin{array}{l}\text { Les agents qui coordonnent le déroulement des diverses cérémonies, de la mise en bière jusqu'à } \\
\text { l'inhumation ou la crémation. } \\
\text { Maîtres de cérémonie ; ordonnateurs ou monteurs de convois. Hôtesses ; téléphonistes ; ven- } \\
\text { deurs ou vendeuses. }\end{array}$ \\
\hline 4 & $\begin{array}{l}\text { Les agents qui concluent directement avec la famille l'organisation et les conditions de la presta- } \\
\text { tion funéraire. } \\
\text { Assistant funéraire; conseiller funéraire ou régleur. }\end{array}$ \\
\hline 5 et 6 & $\begin{array}{l}\text { Les agents qui sont responsables d'une agence, d'un établissement, d'une succursale ou d'un } \\
\text { bureau dans lequel sont accueillies les familles qui viennent conclure des prestations funéraires. } \\
\text { Les gestionnaires d'une chambre funéraire ou d'un crématorium. Les personnes qui assurent la } \\
\text { direction des régies, entreprises ou associations habilitées. }\end{array}$ \\
\hline
\end{tabular}

dans les poignées » est nécessaire à la coordination de l'inhumation. Si le collectif de travail, composé des quatre porteurs et du maître de cérémonie, n'est pas bien coordonnée - si la corde est plus longue d'un côté que de l'autre ou si elle «coince » quand on la retire - la réalisation de la prestation n'apparaîtra pas comme professionnelle. Idem pour la circulation des gens ou la gestion des fleurs.

Ces attentions sont destinées à la fois au mort et aux endeuillés. Elles construisent petit à petit le sérieux de la situation. Car au cours des funérailles, nombreux sont en réalité les moments d'inactivité des agents funéraires. Un effort de contrôle de ses expressions sert, selon Goffman, à se protéger, à protéger les autres participants et à sauver la cérémonie. À défaut d'engagement dans la solennité du moment, "l'illusion de la réalité se brise[rait], la minutie du système social qu'avait créé la rencontre se désorganise[rait] et les participants se sentent[sentiraient] déréglés, irréels, anormaux» (Goffman, 1974, p. 119). La compétence des agents funéraires concerne donc ici l'appropriation de l'espace comme une "scène de théâtre ». Ils ne doivent pas rire. Des compétences apparaissent sur des modalités non verbales et sur le rituel.

Ces savoirs sur la gestion des émotions sont de l'ordre du «travail invisible» (Hochschild, 1983), difficilement évaluable et à la fois individuel et collectif. Même si empiriquement seuls les sujets sont émus, les tactiques de régulation des émotions apparaissent, quant à elles, comme collectives.

D'abord, l'organisation du travail nécessite une division des tâches tenant compte des capacités de chacun; la personnalité, la biographie ou l'état d'esprit des employés sont pris en compte par les directeurs. Par exemple, ils feront en sorte de ne pas envoyer un employé qui vient d'être papa chercher quelque part le corps d'un enfant décédé. Ensuite, le collectif gère les situations délicates par l'humour. Les plaisanteries échappatoires, le rire, la cordialité entre collègues, en effet, relativisent et mettent à distance les épreuves. Enfin, il s'agit de trouver des raisons pour continuer à le faire. Un travail sur les représentations et le rôle social du métier est nécessaire pour que les agents conservent et développent leur estime d'eux-mêmes.

Dans l'équipe de porteurs naît ainsi une " socialisation en acte» qui s'apparente à un apprentissage collectif de la gestion des émotions. Cela interroge la notion de compétence. S'il n'y a pas de «modèle de la compétence » dans les pompes funèbres, nous verrons que cette notion sert de levier à la professionnalisation et à la formation professionnelle. 


\section{GÉRER LES ÉMOTIONS, UNE COMPÉTENCE AU COEUR DE LA PROFESSIONNALISATION}

La gestion des émotions se situe au cœur de la relation de service personnalisée que souhaitent voir se développer le législateur, les clients eux-mêmes et les opérateurs funéraires soucieux de faire valoir leur « compétence». Celle-ci est définie au regard de la satisfaction du client, conçue comme objectif dans la formation professionnelle des opérateurs funéraires. Le nouveau dispositif de validation et de certification intègre ce leitmotiv, ce qui suggère, finalement, qu'une éventuelle «compétence émotionnelle» ne peut se développer que sur la base d'une solide compétence technique.

\section{Une relation de service personnalisée}

Un rappel sur la notion de compétence s'impose. Du point de vue de l'ergonomie, les compétences sont des ressources pour l'action (Weil-Fassina et Pastré, 2004). Proche de la performance, la compétence, au singulier, se voit dans la réussite de l'action en contexte. Elle se distingue de la qualification, qui représente un potentiel reconnu et attribué à un travailleur, parce qu'elle désigne plutôt la capacité de mettre en œuvre celle-ci, de «savoir mobiliser» une «combinaison de ressources» (le Boterf, 1997). Comme la « professionnalité », la compétence - supposée ou avérée d'un professionnel permet qu'on lui accorde sa confiance : elle garantit sa capacité à travailler en toutes circonstances.

L'évaluation des compétences pose problème, « selon la visée opératoire de l'auteur qui l'emploie, d'organisation du travail, de formation de gestion de l'emploi, de négociation de sa reconnaissance...»" (Lichtenberger, 2003). L'émergence de son utilisation et l'évocation d'un «modèle » témoignent d'une transformation sociale des systèmes organisationnels ${ }^{3}$ et l'importance croissante de l'innovation et

\footnotetext{
${ }^{3}$ Transformation qu'il faut néanmoins relativiser tant dans son opposition au modèle de la qualification qu'au niveau de la fréquence de sa mise en place effective dans les entreprises. Lichtenberger, 1999, art. cit. ; Colin T. et Grasser B., 2003.
}

de la flexibilité, ainsi que l'investissement, la coopération et l'autonomie des employés dans la stratégie d'entreprise. Elle crée en tout cas «les conditions d'une prescription des comportements plus que des tâches » (Monchatre, 2003).

Dans les pompes funèbres, l'usage principal de la notion de compétence dans les pompes funèbres correspond, en d'autres termes, à une valorisation de la relation de service personnalisée, ce qui coïncide avec le troisième et dernier temps du « mouvement de la compétence » proposé par Y. Lichtenberger (2003, art. cit.) : après le temps de la revendication de la compétence face au hiatus entre travail réel et travail prescrit et celui du décloisonnement des hiérarchies et des fonctions, succède «la valorisation de produits et services ajustés à la diversité des clients et leurs modes d'usage ».

La compétence en appelle ici à des compromis « entre les trois pôles de l'activité professionnelle : la tâche et l'efficacité de son exécution, l'intérêt pour soi et en particulier pour la préservation de sa santé physique et psychique, les autres et les aspects collectifs du travail » (Gaudard et Weill-Fassina, 1999).

Ces compromis ou arbitrages cognitifs s'insèrent dans la définition de la notion de « compétence de service », qui se révèle de plus en plus importante dans le champ de la formation professionnelle (Éducation Permanente, $\left.\mathrm{n}^{\circ} 137,1998\right)$ et pour de nombreuses organisations (Ughetto et al., 2002). La maitrise de l'interaction de service, plus que de disposition individuelle et naturelle, est une question d'« organisation»; elle est distribuée (Combes, 2002), et résulte d'un processus de coordination qui la consolide en retour.

Replacer le processus de professionnalisation des pompes funèbres dans son contexte institutionnel permet de se convaincre de la «jeunesse» de la profession, ce qui explique encore le flou entourant la notion de compétence dans ce milieu.

Deux décisions politiques majeures ont eu pour objectif de libéraliser l'accès au secteur tout en essayant d'organiser de nouvelles formes de régulations (Trompette et Bossin, 2004). La première, aux conséquences principalement économiques, ouvre progressivement le marché funéraire ; par la loi 93- 
$23^{4}$, le régime de « monopole public » est transformé en un « service public concurrentiel » (Auby, 1997). La seconde modification est d'ordre plus juridique ; par la mise en place, le 9 mai $1995^{5}$, d'un Règlement national des pompes funèbres, deux conditions à l'exercice de la profession sont prévues : la confor- mité aux normes des équipements techniques, et la présentation d'un justificatif de «capacité professionnelle ». Les opérateurs de pompes funèbres doivent en effet attester d'une formation, ou, à défaut, d'une expérience faisant valoir leur capacité (cf. encadré 3 ).

\section{Encadré 3}

\section{Les conditions minimales de certifications et les contenus de la formation professionnelle selon les niveaux (encadré 2) dans le secteur funéraire}

\begin{tabular}{|c|c|c|}
\hline Niveau & Type de certification & Contenu de la formation professionnelle \\
\hline $\begin{array}{c}1 \\
\text { Exemple : } \\
\text { porteur }\end{array}$ & $\begin{array}{l}\text { Attestation de formation } \\
\text { professionnelle de } 16 \mathrm{~h} \\
\text { ou } 12 \text { mois d'expérience } \\
\text { professionnelle. } \\
\text { Certificat d'aptitude } \\
\text { physique de la médecine } \\
\text { du travail. } \\
\text { Copie du permis } \\
\text { de conduire }\end{array}$ & $\begin{array}{l}\text { Hygiène et sécurité, protection : } 2 \mathrm{~h} 30 \\
\text { Psychologie du deuil : } 4 \mathrm{~h} 30 \\
\text { Législation et réglementation funéraires : } 9 \mathrm{~h} \\
\text { Total : } 16 \mathrm{~h}\end{array}$ \\
\hline $\begin{array}{l}2 \text { et } 3 \\
\text { Exemple : } \\
\text { Maître de } \\
\text { cérémonie }\end{array}$ & $\begin{array}{l}\text { Attestation de formation } \\
\text { professionnelle de } 40 \mathrm{~h} \\
\text { ou } 12 \text { mois d'expérience } \\
\text { professionnelle }\end{array}$ & $\begin{array}{l}\text { Législation et réglementation : } 16 \mathrm{~h} \\
\text { Psychologie du deuil : } 8 \mathrm{~h} \\
\text { Protocoles, pratique et symbolique des rites funéraires : } 16 \mathrm{~h} \\
\text { Total : } 40 \mathrm{~h}\end{array}$ \\
\hline $\begin{array}{l}\quad 4 \\
\text { Exemple : } \\
\text { Conseiller } \\
\text { funéraire }\end{array}$ & Idem & $\begin{array}{l}\text { Législation et réglementation : } 40 \mathrm{~h} \\
\text { Prévoyance funéraire et tiers payant : } 16 \mathrm{~h} \\
\text { Information des familles : } 8 \mathrm{~h} \\
\text { Psychologie du deuil, pratique des rites funéraires : } 16 \mathrm{~h} \\
\text { Cas pratiques : } 16 \mathrm{~h} \\
\text { Total : } 96 \mathrm{~h}\end{array}$ \\
\hline $\begin{array}{l}5 \text { et } 6 \\
\text { Exemple : } \\
\text { Responsable } \\
\text { d'agence }\end{array}$ & $\begin{array}{l}\text { Attestation de formation } \\
\text { professionnelle de } 136 \mathrm{~h} \\
\text { ou } 24 \text { mois d'expérience } \\
\text { professionnelle }\end{array}$ & $\begin{array}{l}\text { Idem niveau } 4 \text { plus : } \\
\text { Gestion du personnel : } 20 \mathrm{~h} \\
\text { Gestion comptable : } 20 \mathrm{~h} \\
\text { Total : } 136 \mathrm{~h}\end{array}$ \\
\hline
\end{tabular}

On retrouve dans ce contexte les éclairages qu'apporte la sociologie des professions. Du côté des acteurs, le recours à la nécessité de professionnaliser leur activité vise à «faire reconnaitre leur légitimité et leur compétence» (Labruyère, 2000). Du côté de la branche, la professionnalisation vise un contrôle

\footnotetext{
${ }^{4}$ Loi n ${ }^{\circ} 93-23$ du 8 janvier 1993.

5 Art. L. 2223-20 et suivants du Code Général des Collectivités Territoriales.
}

de l'accès à la profession (Chapoulie, 1974). La formation professionnelle apparaît comme un indice de professionnalisation, ce qui ne veut pas dire pour autant que l'attestation de capacité professionnelle soit forcément gage de compétences professionnelles (Boucheix et Chanteclair, 2000).

Depuis quelques années, la tendance s'accélère pourtant. En 2004, la DGEFP (Délégation générale à l'emploi et à la formation professionnelle) a présenté 
un projet de développement et de validation des compétences des salariés de la branche des services funéraires au CNOF (Conseil national des opérations funéraires) (Cnof, 2004). En 2005, un accord a été signé entre les principaux acteurs de la branche pour permettre "l'accès aux salariés à la formation tout au long de la vie professionnelle et le renforcement de leurs qualifications $»^{6}$. En 2006, les rapporteurs du Sénat ont présenté leur bilan et leurs recommandations et en 2007, le nouveau CQP sera effectif.

Ainsi, depuis 1995, tout nouvel entrant dans les pompes funèbres doit, théoriquement, quel que soit son statut, justifier d'une formation professionnelle certifiant sa capacité à exercer. Mais, en pratique, le problème se pose pour les entrants d'avant 1995. Dans le but de s'assurer du droit à exercer de tous les professionnels, il semble aussi, selon le Rapport 2006, que les mesures de contrôle des préfectures et de l'inspection du travail soient en cause. En outre, s'agissant des organismes de formation, le juriste J.-F. Auby (1997, p. 118) faisait remarquer «il n'y a pas, à l'heure actuelle, de contrôle des pouvoirs publics sur le contenu des stages, les organismes de formation, les personnels en charge des enseignements ».

\section{Un nouveau dispositif de validation et de certification}

Pour les entreprises, la formation professionnelle relève d'un investissement immatériel de développement des compétences d'autant plus important qu'il n'y a pas de formation initiale ou de qualification nécessaires pour entrer dans les pompes funèbres.

Le lien entre la formation professionnelle des pompes funèbres et l'Université est (encore) inexistant. Pour les directeurs au moins, il y aurait pourtant lieu de développer encore plus les modules de formation en sciences humaines et sociales. Elle permettrait d'aller du général au particulier dans les conceptions du travail. Les formations dispensées dans les établissements privés intègrent ces questions, mais de la théorie à la compétence en acte, il est difficile de s'assurer de l'harmonisation des compétences requises préalable à leur transférabilité.

${ }^{6}$ Accord du 23 juin 2005 inscrit à la Convention collective des pompes funèbres.
Car au-delà du panel d'offre de formations, le problème vient d'abord du fait que tous les directeurs ne sont pas convaincus de leur intérêt. Alors que certaines entreprises jouent le jeu et prônent la formation comme une «stratégie», voire comme un « développement personnel» (entretien directeur et formateur, 47 ans), d'autres considèrent que l'« apprentissage sur le tas » et l'expérience suffisent'. De plus, le recours à la formation du personnel et à la «gestion des compétences 》 est plus difficile pour les petites entreprises (Collin et Grasser, 2003, art. cit.).

Cependant, il y a encore quelques années, l'attestation de capacité professionnelle était attribuée à quiconque suivait le stage de formation. Pour donner valeur diplômante à la formation, un premier CQP «métiers du funéraire » sur la base d'une vérification des connaissances minimum vérifiait si le postulant au CQP savait respecter la réglementation. Le CQP «nouvelle formule» prolonge cet effort. Ce qui se faisait restera en place, et correspondra à une première Unité de valeur (UV) sanctionnée par la réussite à un Questionnaire à choix multiples. Cinq autres UV ont été rajoutées. Celles-ci pourront être reconnues sur la base d'une Validation des acquis de l'expérience (VAE) par un examen oral devant deux membres de jury extérieurs à l'entreprise. La formation pourra être dispensée au cours de stages au sein des différents organismes - rattachés aux réseaux d'entreprises ou aux franchises - ou par l'intervention sur les lieux de travail d'un formateur extérieur. L'idée des auto-confrontations croisées (Clot et alii, 2001) serait à tester. On peut noter que, dans la foulée de ce nouveau CQP pour les assistants funéraires, un CQP pour les porteurs est en projet.

Les critères utilisés en VAE ( $c f$. encadré 4) constituent un premier usage de la notion de compétence dans le secteur, celui des formateurs. Voyons également d'autres points de vue.

\section{La compétence technique, support de la compétence émotionnelle}

Les acteurs du funéraire se rejoignent dans l'intention de valoriser l'image de leur métier. Ils considèrent que «l'opinion» réduit trop souvent leur

${ }_{7}$ J'ai travaillé dans deux entreprises bien différentes sur ce point. 


\section{Encadré 4 \\ Un nouveau dispositif de validation et de certification, le Certificat de qualification professionnelle (CQP) assistant funéraire 2007}

Le nouveau CQP peut être considéré comme le premier référentiel de compétences dans la profession. Il est organisé autour des six unités de valeur suivantes:

Unité 1: Organiser les obsèques en mettant en œuvre la réglementation.

Unité 2: Accueillir et mener un entretien avec la famille et les proches.

Unité 3 : Concevoir, expliquer et animer une cérémonie d'obsèques (développement d'une cérémonie, rites et sens des pratiques funéraires).

Unité 4 : Vendre des produits et services funéraires (techniques de vente, connaissance des produits et services, établissement d'un devis, connaissance des coûts, modes de financement et de règlement).

Unité 5: Adapter son comportement à la déontologie de la profession et aux situations relationnelles avec la famille en deuil (communication interpersonnelle, psychologie et sociologie du deuil, déontologie de la profession, notions de qualité et de certifications de la qualité).

Unité 6: Organiser son activité au sein d'une équipe et traiter les informations nécessaires (techniques d'organisation et de planification, risques professionnels et mesures de sécurité, techniques de rédaction, d'expression écrite, informatique, bureautique, progiciels de gestions spécifiques).

Source : « Circulinfo », lettre d’information du réseau Funéplus, août 2006.

activité au profit engendré dans les circonstances du deuil et néglige la difficulté de leur tâche. «Dès qu'on parle de pompes funèbres, on parle de vautours, il y a des images... de rapaces! » (responsable d'agence, 53 ans).

Les directeurs ont des points de vue différenciés mais complémentaires sur ce qu'est «un bon professionnel». Certains mettent en avant des compétences techniques et d'autres des qualités «psychologiques »: «quand on reçoit les gens ici au bureau... c'est un grand mot mais... faut être un peu psychologue» (directeur, 62 ans). Pour beaucoup, les qualités principales dont doit disposer un bon opérateur sont l'empathie, l'écoute et... la force de vente.

Dans l'accomplissement des cérémonies, il faut être « fort »... mais pas trop : «faut pas être fermé, euh, oui et non, on n'est pas étanche à ce qui se passe [...] Faut être très discret mais très présent " (directeur, 36 ans). Cet extrait d'entretien à deux voix résume la difficulté de problématiser le triangle sensibilité-expérience-formation :

Le premier: "Il faut de la douceur, de l'accompagnement, ça s'apprend, ça s'apprend, enfin ça s'apprend pas. » Le second : "C'est l'expérience... la sensibilité, il faut avoir de la sensibilité intérieure et puis... ça s'fait. Faut pas faire ça si on n'en a pas... » Le premier : "Vous savez, dans notre profession, il va y en avoir qui en auront "rien à faire" parce qu'ils n'auront aucun sentiment. S'ils mettent une intonation qui va pas, la famille ça va pas leur plaire. Même si le service est fait standard.» Moi : «Alors la sensibilité fait partie de la compétence. » Le premier: «Ah oui.» Le second acquiesce (Entretien avec deux cogérants, 36 et 33 ans).

Un bon opérateur est aussi celui qui sait gérer son personnel, notamment en lui faisant découvrir l'univers symbolique de son activité : «S'il n'y a pas un professionnalisme derrière pour dire quelle est la signification de votre geste, eh bien ça sert à rien. »

Dans leur logique d'embauche, les directeurs distinguent les compétences requises pour leurs employés permanents - la polyvalence et la « souplesse »des compétences de leur personnel «intermittent» (porteurs), qui doivent surtout «être présentables» (discrétion, tenue vestimentaire correcte). Mais ils évaluent aussi son «équilibre psychologique». La mise en lien des compétences requises suggère en effet la capacité d'articuler les dimensions techniques et émotionnelles de son travail. 
La notion de compétence est ainsi brandie dans le but de satisfaire la famille en respectant son émotion et la solennité des circonstances. D'ailleurs, pour connaître le point de vue des familles, difficilement accessible, certaines entreprises mettent en place un questionnaire de satisfaction et ont ainsi un retour, le plus souvent positif, sur leur prestation.

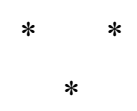

Gérer ses émotions, comprendre les émotions des endeuillés, agir dans une ambiance émotionnelle, sont autant d'éléments qui peuvent être considérés comme des compétences de service à condition de prendre en compte la dimension collective et de problématiser l'usage implicite de la notion de compétence relationnelle dans la formation professionnelle des agents funéraires. Capacité de réflexion, engagement dans l'action, coordination sociale, par exemple, sont des éléments dans lesquels interviennent les émotions et qui sont souvent cités comme des compétences professionnelles. Penser les émotions peut ainsi servir la professionnalisation des pompes funèbres, la formation professionnelle des agents et l'amélioration de la relation de service d'un point de vue pratique. Les émotions sont des « compétence(s)» des pompes funèbres dans un double sens. Elles sont à la fois l'objet de l'«habilitation» sociale de prise en charge des endeuillés dévolue, de plus en plus, aux pompes funèbres, et du «travail invisible» réalisé en situation par les agents funéraires.

En conclusion, deux questionnements méritent d'être abordés, celui de l'enjeu de la place de la « gestion des émotions » dans l'élaboration des référentielscompétence. Et celui, théorique, de la notion $\mathrm{d}^{\prime}$ « intelligence émotionnelle ».

Dans la mise en œuvre du CQP «assistant funéraire », à partir de quand quelqu'un qui ne saurait pas bien gérer les émotions serait-il jugé incompétent? Normaliser l'attitude attendue des travailleurs, est-ce formater l'individu ou développer la compétence ? Il y a lieu de se poser la question si l'on considère la notion de savoir-être comme «un instrument de légitimation des jugements subjectifs portés sur les individus» (Sulzer, 1999). Il semble que cette question se trouve en filigrane de la discussion entre J.-P. Durand (2000) et P. Zarifian (2000). Pour le premier, la mobilisation de la subjectivité de tous les salariés correspond à une nouvelle forme de contrôle social du travail et d'«achat de la personnalité », alors que pour le second, ces dimensions ne peuvent rester privées, «non publiques, non négociées, non discutées ». Mais comment évaluer la qualité de la gestion émotionnelle ? La notion de " savoir-être », utilisée trop souvent pour définir les compétences professionnelles, semble d'autant plus devoir être remise en question que le CQP n'intègre pas le travail collectif.

Que comprendre, en effet, sous le terme d'intelligence ou de compétence émotionnelle ? La notion d'intelligence émotionnelle, en vogue aux ÉtatsUnis et dans le monde de la gestion, a surtout consisté à mettre en avant les capacités du leader ou à expliquer ses performances personnelles et/ou celles de l'organisation par ses capacités à prendre conscience de ses propres émotions et celles d'autrui et à les réguler (Goleman, 1995). Or, comme le constate J.-F. Chanlat (2002), le courant de l'intelligence émotionnelle naturalise les émotions et néglige le contexte socioculturel des relations sociales, les relations de pouvoir, ou les questions d'éthique. L'individualisation de la notion d'intelligence émotionnelle est un écueil possible, mais non nécessaire, à la problématisation des compétences « émotionnelles ». Celle-ci nécessite d'interroger les liens minutieux entre travail individuel et collectif, émotions et situations. 
Auby J.-F. (1997), Les obsèques en France, PUF, QSJ, Paris.

Boucheix J.-M. et Chanteclair A. (2000), « Quand la certification pourrait ne pas voir la compétence », Formation Emploi, n ${ }^{\circ}$ 69. pp. 37-52.

Cefaï D. (coord.) (2003), L'enquête de terrain, La Découverte/MAUSS, Paris.

Chanlat J.-F. (2002), «Émotions, organisation et management: une réflexion critique sur la notion d'intelligence émotionnelle », Travailler, $\mathrm{n}^{\circ} 9$, pp. 113-132.

Chapoulie J.-M. (1974), « Sur l'analyse des groupes professionnels", Revue française de sociologie, $\mathrm{n}^{\circ} 14$, pp. 86-114.

Clot Y., Faïta D., Fernandez G. et Scheller L. (2001), «Entretiens en autoconfrontation croisée: une méthode en clinique de l'activité », Éducation Permanente, $\mathrm{n}^{\circ} 146$, pp. 17-25.

Colin T. et Grasser B. (2003), «La gestion des compétences : vraie innovation ou trompe-l'œil ?», Colloque DARES Travail et relations sociales en entreprise.

Combes M.-C. (2002), «La compétence relationnelle : une question d'organisation », Travail et Emploi, $\mathrm{n}^{\circ}$ 92, pp. 5-18.

Damasio A. (1995), L'erreur de Descartes, la raison des émotions, Odile Jacob, Paris.

Desjours C. (1993), Travail et usure mentale, Bayard, Paris.

Dumouchel P. (1995), Émotions : essai sur le corps et le social, Synthélabo, Paris.

Durand J.-P. (2000), «Les enjeux de la logique compétence », Gérer et comprendre. Annales des Mines.
Éducation Permanente (1998), n 137.

Gaudard C. et Weill-Fassina, A. (1999), «Activités de travail et dynamique des compétences. » Formation Emploi, 67, pp. 47-62.

Goffman E. (1968), «La relation de service» in Asiles, Minuit, Paris.

Goffman E. (1974), Les rites d'interaction, Minuit, Paris.

Goleman D. (1997), L'intelligence émotionnelle. Comment transformer ses émotions en intelligence, Robert Laffont, Paris.

Grosjean V. et Van de Weerdt C. (2003), «Approche psycho-ergonomique du bien-être au travail : le cas des centres d'appel », Épique.

Guibe-Hubert N. (2002), « Présentation des divers métiers du funéraire et de leurs risques professionnels », www.federationsantétravail.org

Hughes E. (1976), « Le drame social du travail», trad. fr., Actes de la recherche en sciences sociales, $\mathrm{n}^{\circ} 115,1996$.

Hochschild A. (1975), « Travail émotionnel, règles de sentiments et structure sociale », trad. fr., Travailler, $\mathrm{n}^{\circ} 9$, pp. 19-50.

Hochschild A. (1983), The Managed Heart, California Press, Berkeley.

Jeantet A. (2002), «L'émotion prescrite au travail », Travailler, $\mathrm{n}^{\circ}$ 9, pp. 99-112.

Labruyère C. (2000), « Professionnalisation : de quoi parlent les chercheurs, que cherchent les acteurs ?», Formation Emploi, n ${ }^{\circ}$ 70, pp. 31-42.

Le Boterf G. (1997), Compétence et navigation professionnelle, Éditions d'Organisation, Paris. 
Leplat J. (1997), Regards sur l'activité en situation de travail. Contribution à la psychologie ergonomique, Paris, Presses Universitaires de France.

Lichtenberger Y. (1999), « Compétence, organisation $\mathrm{du}$ travail et confrontation sociale », Formation Emploi, ${ }^{\circ}$ 67, pp. 93-107.

Lichtenberger Y. (2003), «Compétence, compétences ", in Encyclopédie des ressources humaines, Vuibert, Paris.

Livet P. (2002), Émotions et rationalité morale, Presses Universitaires de France, Paris.

Loriol M. et Weller J.-M. (2002), « La prescription des émotions pour les infirmières hospitalières et les volontaires de AIDES : contrôle de soi et juste distance au malade », Self, pp. 316-321.

Ministère de l'Emploi, de la Cohésion sociale et du Logement, Convention collective nationale des Pompes funèbres, accord du 23 juin 2005, brochure $\mathrm{n}^{\circ} 3269$.

Monchatre S. (2003), « Management des compétences et construction des qualifications », Bref-Cereq, $\mathrm{n}^{\circ} 201$.

Olry P. (2005), «Distance thérapeutique et corps à corps. L'exemple du traitement de la douleur en massokinésithérapie », Éducation Permanente, $\mathrm{n}^{\circ} 165$.

Paperman P. et Ogien R. (dir.) (1995), « La couleur des pensées: sentiments, émotions, intentions", Revue Raisons pratiques, vol. 6, EHESS, Paris.

Sueur J.-P. et Lecerf J.-R. (2006), Rapport d'information sur le bilan et les perspectives de la législation funéraire, $\mathrm{n}^{\circ} 372$, Sénat.

Shott S. (1979), "Emotion and social life : A symbolic interactionist analysis", American Journal of Sociology, 84, (6), pp. 1317-1334.

Soares A. (2002), «Les émotions dans le travail», Travailler, $\mathrm{n}^{\circ} 9$.

Sulzer E. (1999), «Objectiver les compétences d'interaction. Critique sociale du savoir être», Éducation Permanente, $\mathrm{n}^{\circ} 140$.
Thoits P. (1990), "Emotional deviance: Research Agendas", in Kemper T. (dir.), Research Agendas in the Sociology of Emotions, State University Press, New York, pp. 180-206.

Trompette P. et Caroly S. (2006) « De la compétence de service aux compétences de coordination et d'orchestration. Autour du conseiller funéraire», Pistes, vol. 8/1.

Trompette P. et Boissin O. (2002), Les services funéraires. Du monopole public au marché concurrentiel, Rapport d'étude pour la DARES, ministère des Affaires sociales, du Travail et de la Solidarité, convention $\mathrm{n}^{\circ} \mathrm{E} 322,2000-2002$.

Trompette P. et Boissin O. (2004-a), «La famille face au marchand de pompes funèbres: analyse d'une situation de confiance sous contrainte » in Mangematin V. \& Thuderoz C., Des mondes de confiance, un concept à l'épreuve de la réalité sociale, CNRS éd., Paris.

Trompette P. et Boissin O. (2004-b), «La libéralisation des services funéraires, des régulations qui se cherchent », Économie et Sociétés, n 24.

Ughetto P. (2002), « Compétence de service : état des lieux d'une problématique », document IRES $\mathrm{n}^{\circ}$ 02-03.

Ughetto P. Besucco N., Tallard M., du Tertre C. (2002-b), La relation de service : un nouveau modèle de travail en tension, Rapport ACI Transformations $\mathrm{du}$ travail, performance économique et statut de l'emploi.

Ughetto P., (2004), «Au service d'un public: un détour par Halbwachs et Goffman », document IRES $\mathrm{n}^{\circ}$ 04-09.

Weil-Fassina A. et Pastré P. (2004), « Les compétences professionnelles et leur développement», in Falzon P. (dir.) Ergonomie, PUF, Paris.

Zarifian P. (2000), «Sur la notion de compétence, réponse à J.-P. Durand» Gérer et comprendre. Annales des Mines. 


\section{Résumé}

\section{La gestion des émotions aux pompes funèbres, une compétence reconnue?}

Julien Bernard

Cette contribution cherche à caractériser les compétences professionnelles des agents des pompes funèbres, à préciser le rôle que jouent les émotions dans leur activité de travail et les relations entre compétences et émotions. À partir d'une observation participante, nous analysons la prise en compte des émotions dans le collectif de travail, leur régulation et leurs modes de reconnaissance comme compétence. La coordination du travail, les savoirs professionnels et les techniques de gestion émotionnelle apparaissent en effet au cours d'interactions complexes dont la maîtrise est l'enjeu de la professionnalisation du secteur et de la formation.

Mots-clés

Pompes funèbres, compétence, savoir-être

Journal of Economic Literature: J 24 